Horizons philosophiques

\title{
Construction et structure dynamique des théories physiques
}

\section{Yvon Gauthier}

Volume 2, numéro 2, printemps 1992

Philosophie et sciences : du concept au réel

URI : https://id.erudit.org/iderudit/800895ar

DOI : https://doi.org/10.7202/800895ar

Aller au sommaire du numéro

\section{Éditeur(s)}

Collège Édouard-Montpetit

\section{ISSN}

1181-9227 (imprimé)

1920-2954 (numérique)

Découvrir la revue

\section{Citer cet article}

Gauthier, Y. (1992). Construction et structure dynamique des théories physiques. Horizons philosophiques, 2(2), 51-63.

https://doi.org/10.7202/800895ar d'utilisation que vous pouvez consulter en ligne.

https://apropos.erudit.org/fr/usagers/politique-dutilisation/ 


\section{Construction et structure dynamique des théories physiques*}

\section{Présentation}

Le texte "Construction et structures dynamiques des théories physiques" s'inspire de la problématique d'une logique interne des discours scientifiques, en particulier des mathématiques et de la physique. L'hypothèse générale d'une logique locale, chaque fois singularisée par son objet, va à l'encontre d'une tradition philosophique qui a supposé que la logique était universelle, sorte de forme vide qui insufflerait les langages et les théories dans l'abstraction des principes régulateurs de la raison. C'est plutôt la matière concrète ou le matériau (ou encore le contenu) qui détermine la forme qu'un discours doit prendre. Les mathématiques et la logique intuitionnistes nous ont appris que la logique de l'infini différait de la logique du fini et la mécanique quantique a montré que la logique des événements quantiques n'était plus la logique (booléenne) des situations ordinaires. Si on peut parler d'une logique quantique, c'est que l'interaction entre un système observé et un système observateur entraîne une modification de la théorie de la mesure qui à son tour influe sur la structure

* Ce texte constitue le chapitre 2 d'un ouvrage intitulé La Logique interne des théories physiques à paraître au cours de l'année 1992 chez J. Vrin, coll. “Analytiques". 
fine de la théorie. Même la théorie des probabilités sousjacente doit être révisée. On arrive ainsi à la formulation d'une théorie générale de la logique interne qui définit une hiérarchie de logiques étrangère à la vision impériale d'un discours unificateur.

0.

La notion de théorie physique que nous voulons cerner est assurément plus complexe que celle que $P$. Duhem $^{1}$ a pu circonscrire. Poincaré et Hilbert étaient plus sensibles à la dimension mathématique (l'appareil mathématique) qu'à l'architecture globale de la théorie physique. Plus près de nous, les travaux de J.D. Sneed ${ }^{2}$ et Stegmüller (voir là-dessus J. Leroux ${ }^{3}$ ), s'ils s'inspirent de la thèse ou de l'hypothèse de Duhem selon laquelle une théorie possède une structure globale qu'il n'est pas possible d'invalider par une expérience cruciale, ne font que proposer un schéma général, celui du prédicat ensembliste, pour rendre compte de la structure logique des théories physiques. L'idée du prédicat ensembliste empruntée à Suppes consiste simplement à définir l'appartenance d'une théorie à un univers ensembliste de la même façon qu'on définit une structure par l'appartenance à un univers ensembliste (d'individus, de fonctions et de prédicats) dans la théorie élémentaire des modèles. On ne produit alors qu'un cadre abstrait, une structure logico-mathématique nue qui n'est que le reflet ensembliste (naiif) de la théorie physique la mécanique classique des particules, par exemple.

1. La Théorie physique, son objet et sa structure, Paris, Chevalier et Rivière, 1906.

2. The Logical Structure of Mathematical Physics, Dordrecht-Holland, Reidel, 1971.

3. La Sémantique des théories physiques, Ottawa, PUO, 1988. 
L'entreprise de G. Ludwig 4 est plus ambitieuse : fonder la théorie physique, en particulier la mécanique quantique, sur la théorie axiomatique des ensembles. Ici la notion de structure est celle de Bourbaki et elle a perdu sa naïveté mathématique. Sans entrer dans les détails de la construction de Ludwig, donnons quelques éléments qui éclaireront le projet. Tout d'abord, Ludwig distingue trois composantes fondamentales d'une théorie physique que l'on peut illustrer ainsi

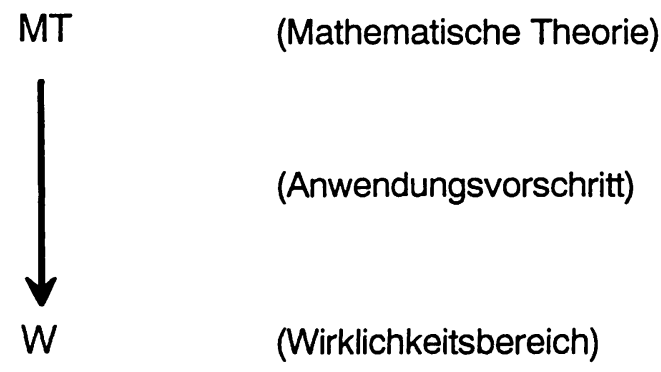

La théorie mathématique est injectée dans un domaine de réalité qui contient, en plus des objets propres de la théorie physique, les objets réels d'un domaine fondamental ("Grundbereich») qui peuvent appartenir à une autre théorie physique, mais qui d'une certaine façon déterminent le contenu objectif de la théorie physique, tout en ne pouvant être déterminés entièrement que par cette dernière; ce texte réel («Realtext») est le sol sur lequel on édifiera la structure axiomatique.

4. Deutung des Begriffs «Physikalische Theoriew und axiomatische Grundlegung der Hilbertraumstruktur der Quantenmechanik durch Hauptsätze des Messens, Berlin - Heidelberg - New-York, Springer - Verlag, 1970. 
Chez N. Bourbaki ${ }^{5}$, on suppose qu'on a une base composée de trois ensembles $E, F, G$ distincts qui génèrent d'autres ensembles par les opérations d'ensemble des parties et de produit. Une espèce de structure dans une théorie T plus forte que la théorie des ensembles comprendra ainsi des ensembles de base principaux, au besoin des ensembles de base auxiliaires, une typification qui a pour rôle de caractériser l'espèce de structure par un schéma de construction (d'échelon, c'est-à-dire d'ordre de génération des ensembles) et enfin un axiome qui définit l'espèce de structure. Une structure d'espèce $T$ est donc définie sur une base $E, F, G$ par le type des propriétés ou parties d'un ensemble $M$ d'une échelle d'ensembles donnée. C'est ainsi qu'on a les structures de groupe, d'ensemble ordonné, d'espace topologique, etc. Les trois grandes catégories de structures sont les structures-mères, structures d'ordre, structures algébriques et structures topologiques, mais ce qu'on doit retenir ici de la construction bourbakiste, c'est la notion d'échelle d'ensembles et de hiérarchie de structures. La hiérarchie bourbakiste n'est pas sans rappeler la structure cumulative des rangs de la théorie axiomatique des ensembles (due à Zermelo et von Neumann surtout) ${ }^{6}$.

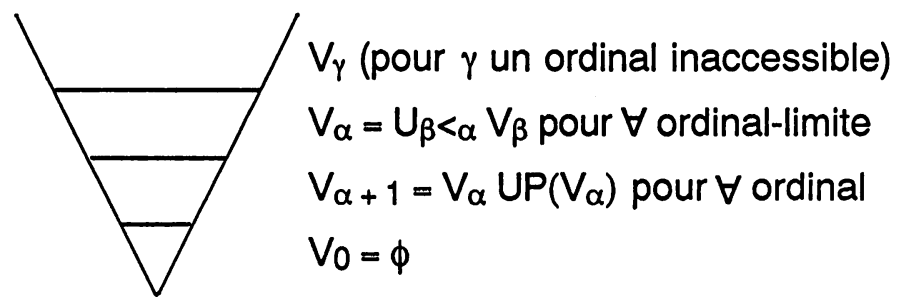

5. Théorie des ensembles, Paris, Hermann, 1970.

6. Y. Gauthier, Fondements des mathématiques. Introduction à une philosophie constructiviste, Montréal, PUM, 1976. 
La structure cumulative est engendrée ici par les deux opérations d'ensemble des parties et union. L'analogie s'arrête là, puisque les espèces de structure s'arriment dans des théories plus fortes que la théorie des ensembles, une structure d'ensemble restant la plus pauvre de toutes les structures. Quel est l'effet de cette construction bourbakiste sur Ludwig?

Force est d'admettre que si l'axiomatisation de Ludwig est plus riche que la reconstruction de Sneed, on n'a encore qu'un squelette, même s'il est plus charnu. L'appareil bourbakiste n'a servi qu'à préparer le texte réel («Realtext"), il ne rend pas compte de la structure de la théorie physique et l'axiomatisation de la structure de l'espace de Hilbert n'a rien de spécialement bourbakiste 7 . En tout cas, I'habillage ensembliste, ici comme ailleurs, n'est rien d'autre qu'une mise en forme qui ne participe pas de la logique interne de la théorie physique. Rien n'interdit, par exemple, de songer à une formulation catégorique (en termes de théorie des catégories) ou toposique (en termes de théorie des topoi) des théories physiques.

\section{2.}

Mais qu'en est-il de la logique interne de ces mêmes théories physiques? Plutôt que d'en discuter abstraitement, je veux mettre à profit deux exemples que je tire de Karl Popper : ces deux exemples se trouvent dans Quantum Theory and the Schism in Physics ${ }^{8}$. Le premier exemple touche à l'interprétation d'Everett en Mécanique Quantique (MQ) et constitue une addition récente (datée de 1981, p. 89-95). L'interprétation d'Everett, qui remonte à la fin

7. E. Scheibe, "Ein Vergleich der Theoriebegriffe von Sneed und Lugwig", Erkenntnis und Wissenschaftstheorie, Wien, éd. P. Weingartner and H. Czermak, 1983, p. 371-383.

8. Vol. 2, Totowa, N.J., éd. W. Bartley III, Roman and Littlefield, 1982. 
des années cinquante, a connu un certain succès à cette époque : elle consistait à supposer que la fonction d'onde dans l'équation de Schrödinger se ramifiait universellement, c'est-à-dire qu'elle prenait toutes ses valeurs et qu'elle engendrait ainsi une multitude d'univers, de multivers, dont nous n'étions pas conscients (pas plus que nous ne sommes conscients de la rotation de la Terre, dit Everett, et pourtant elle tourne, disait l'autre). Popper écrit que l'interprétation ne tient pas puisqu'elle contredit les lois dynamiques de la physique, la solution d'Everett ne respectant pas la symétrie du renversement de la direction du temps, par exemple. Popper ajoute que l'interprétation, par là, n'est pas isomorphe au formalisme de la théorie, ce que prétendait Everett.

Je voudrais montrer à partir de cet exemple qu'on peut aller beaucoup plus loin et refaire ici ma courte démonstration de l'inconsistance de la thèse d'Everett que l'on trouvera ailleurs ${ }^{9}$. La ramification universelle de la fonction d'onde donne un ensemble de cardinalité $2^{\text {*o }}$ (ce que semble remarquer Popper dans une note p. 93 du texte précité), puisque la fonction d'onde prend ses valeurs dans $R$ (ensemble des nombres réels) ou dans $C$ (ensemble des nombres complexes) et que l'on quantifie universellement; mais il n'y a certainement pas plus de ¿0 résultats de mesure et même s'il y avait $\kappa_{0}$ observateurs, $\kappa_{0}+\kappa_{0}=\kappa_{0}$ ou encore $\aleph_{0} \cdot \aleph_{0}=\aleph_{0}$ et il n'y a pas de bijection entre $\aleph_{0}$ et $2^{\text {No; }}$ ce qui montre l'inconsistance de l'interprétation d'Everett et du même coup invalide sa thèse de l'isomorphisme entre le formalisme mathématique et son interprétation physique.

La contradiction apparaît dans la théorie des ensembles, dont Everett ne conteste jamais la validité. Je pose

9. Y. Gauthier, Théoritiques. Pour une philosophie constructiviste des sciences, Longueuil, Le Préambule, 1982, p. 76; "Quantum mechanics and the local observer", The International Journal of Theoretical Physics, no 22 (1983), p. 1141 à 1152. 
la question : une contradiction mathématique n'est-elle pas plus sérieuse qu'une contradiction au sein de la théorie physique? Je suppose que l'ordre de la consistance va dans le sens suivant :

\section{logique $\Rightarrow$ mathématique $\Rightarrow$ physique $\Rightarrow$ philosophie}

Ainsi une contradiction au niveau logique a des répercussions à tous les autres niveaux, alors qu'une contradiction philosophique n'est qu'une auto-affection. II y a d'autres critères que la consistance pour les théories scientifiques en général et physiques en particulier, mais je ne m'intéresse ici qu'à la consistance interne d'une théorie. Le choc en retour d'une impossibilité philosophique peut cependant entraîner une révision de la logique et c'est ce que nous allons voir avec notre deuxième exemple qui semble affecter la validité de la logique classique : c'est l'inégalité de J.S. Bell10.

Reprenant le paradoxe d'Einstein-Podolsky-Rosen sur l'incomplétude de la $M Q$ vieux de 1935, Bell montre que les théories réalistes à paramètres cachés, comme la théorie de David Bohm, entraînent une inégalité qui n'est pas compatible avec les prédictions de la $M Q$. Cette inégalité est liée aux mesures de probabilité des composantes de spin $\mathrm{A}^{+}, \mathrm{B}^{+}, \mathrm{C}^{+}$d'un nombre $\mathrm{n}$ de particules

$$
n\left[A^{+}, B^{+}\right] \leq n\left[A^{+}, C^{+}\right]+n\left[B^{+}, C^{+}\right] \text {. }
$$

Des expériences ont été conçues récemment pour tenter de confirmer cette inégalité et ainsi démontrer l'invalidité de la MQ. Rappelons les expériences spectaculaires d'Aspect qui ont eu un résultat négatif : les expériences sont en accord avec les prédictions de la $M Q$, c'est-à-dire que l'inégalité n'est pas valide. 
Popper écrit dans sa Préface de 1982 au Postcript "On a Realistic and Common Sense Interpretation of Quantum Theory" :

Je dois admettre que les résultats de ces tests m'ont surpris. Lorsque pour la première fois j'ai entendu dire que John $F$. Clauser et Abner Shimony avaient l'intention de vérifier le théorème de Bell, je m'attendais à ce que leurs résultats réfuteraient la théorie quantique. Mais il s'est avéré que mon appréhension était fausse, puisque la majorité des tests prouvèrent le contraire.

Et Popper suggère de ressusciter l'action à distance (et son médium, l'éther) dans l'interprétation de Lorentz pour aller à l'encontre du principe de localité ou séparation spatiale exigé par la Relativité Restreinte (RR) et que les théories réalistes de variables cachées ne peuvent admettre en vertu de l'inégalité de Bell - Bohm parlera alors de "wholeness" et d'Espagnat de non-séparabilité du réel physique. II s'agit ici de savoir si une thèse philosophique, le réalisme "commun" de Popper, est assez forte pour répudier un principe physique dont la validité semble assurée, la causalité relativiste ou l'invariance de Lorentz. Le résultat physique ici semble en contradiction avec la philosophie réaliste. Je pose la question : est-ce que l'exigence philosophique est plus grande que la validité des lois physiques? Je dirai qu'il est plus simple (et plus sage) d'abandonner le réalisme plutôt que la Relativité Restreinte; si j'adopte une logique plus forte, c.-à-d. plus exigeante, la logique constructiviste de la négation locale et du complément local qui n'est plus booléenne, c'est-à-dire qui n'obeit plus à loi $\neg \neg a=a$, alors je peux dire que la mesure, c'est-à-dire la relation ou l'interrelation (ou l'interaction) du système observé et du système observateur (ou de l'appareil de mesure) ne conserve pas les mesures de probabilité sur les sous-espaces orthogonaux de l'espace de Hilbert" ou encore "Ouvre" l'ensemble des sous-

11. Gauthier, "Quantum mechanics...". 
espaces, ce qui entraîne la non-orthocomplémentation et une logique non booléenne. Si le réalisme est répudié ici, on ne tombe pas pour autant dans l'idéalisme : le constructivisme est aussi empirique que le plus béat des réalismes. On sait que certaines notions et certaines théories mathématiques, la notion d'espace topologique et la théorie des topoi en géométrie algébrique où un topos est une généralisation de la notion d'espace topologique, obéissent à une logique intuitionniste. Pourquoi la théorie "constructivisée» des espaces de Hilbert n'obéirait-elle pas à une telle logique, si, par ailleurs, la situation physique où l'on suppose un observateur local l'exige? Popper pensait que la physique pouvait se passer de la notion d'observateur. Je demande : est-il préférable de se passer de la Relativité Restreinte plutôt que de l'observateur local? Remarquez que j'obtiens une théorie consistante de la MQ et de la RR avec le formalisme de l'observateur local que je propose. Mais je laisse Popper ici et j'aborde mon thème général. Je note cependant que ce n'est pas la première fois que Popper se trompe, c'est peut-être pour cette raison qu'il a conçu le falsificationnisme...

3.

Je conçois une théorie physique comme un quintuplet $P=\left\langle A_{p}, M_{p}, \varphi, T_{p}, \psi\right\rangle$ où $A_{p}$ est l'appareil logico-mathématique (ou analytique) ou encore l'ensemble des structures logico-mathématiques de la théorie, $\mathrm{M}_{\mathrm{p}}$ la classe des modèles ou interprétations visées (qui n'ont pas de structure ensembliste), $\varphi$ l'homomorphisme ou la classe des hom entre $A_{p}$ et $M_{p}, T_{p}$ étant l'ensemble constitué par la theoria mundi, c'est-à-dire toute la physique, toutes les théories physiques et extra-physiques qui forment le tissu théorique premier d'une théorie physique particulière Ludwig $^{12}$ appelle Tp le "Grundbereich», le domaine fonda-

12. Deutung des... 
mental des notions, concepts, objets communs à toute théorie physique ou à une classe de théories physiques. Un métaphysicien a ici beau jeu et peut mettre ses vers dans cette pomme du savoir qui est faite pour être croquée. Le morphisme injecte $M_{p}$ dans $T_{p}$ et nous avons le schéma suivant :

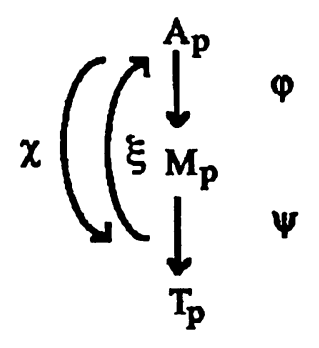

avec $\chi$ comme composition des morphismes $\varphi$ et $\psi$ qu'on peut rendre plus explicite dans le diagramme commutatif suivant :

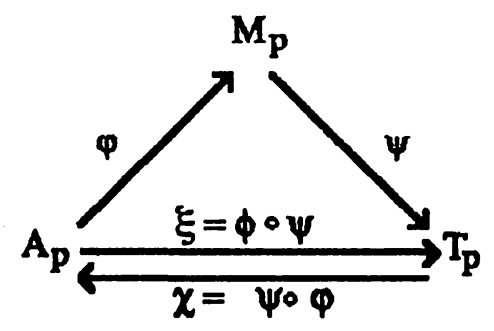

Nous n'avons plus, bien entendu, de règles de correspondance, mais un réseau complexe de relations ou de morphismes qui se composent entre eux : ce qui montre que la théorie physique constitue un tout organique, une structure dynamique.

La double flèche entre $A_{p}$ et $T_{p}$ ne signifie pas qu'il s'agit d'un isomorphisme, mais plutôt d'influences réciproques de l'appareil analytique et de la conception du monde ou theoria mundi, p. ex. équations différentielles partielles et déterminisme chez le dernier Einstein. Le choix de l'appareil analytique influe davantage sur la conception du 
monde, dans la mesure où la théorie logico-mathématique est première dans l'ordre des raisons, de la logique à la philosophie. Mais c'est sur la structure réticulée ou le système holiste des relations entre appareil analytique, modèle ou interprétation physique et théorie du monde qu'il faut mettre l'accent en notant que le poids relatif des éléments ou sommets du réseau varie pour chaque théorie. La dynamique des théories en dépend, en l'absence de toute considération historique sur l'évolution de la théorie, les changements conceptuels ou encore les révolutions scientifiques, si tant est qu'il faille en tenir compte. Mon point de vue est anhistorique dans le contexte d'une logique interne des théories physiques, mais une analyse plus ambitieuse pourrait sans doute montrer que l'unification des théories antérieures est la seule logique du développement historique de la physique moderne, de l'électromagnétisme de Maxwell et de la mécanique statistique aux théories unifiées contemporaines. Même la mécanique newtonienne comme cas-limite de la mécanique quantique s'intègre dans ce schéma unificateur qui devient l'unique facteur de progrès, au-delà et en dépit des prétendues révolutions scientifiques. Cet idéal d'unité ne renvoie pas cependant à l'intégrité de la nature, mais bien plutôt à la solidarité des pratiques et à la communauté des savoirs. Physique pure et physique appliquée ou expérimentale, théorie et technologie ne font qu'un : un bel exemple en est la supraconductivité qui n'est pas possible sans la mécanique quantique, pas plus que ne l'est la microscopie électronique ou encore le laser. Les théories de grande unification, pour extravagantes qu'elles soient, répondent du même idéal, et malgré les professions de foi réalistes des praticiens qui sont souvent éloignés des préoccupations fondationnelles, c'est l'activité théorique et ses retombées pratiques qui fabriquent le tissu unitaire de la science. De part en part, la science, la physique éminemment, est activité de construction avant d'être étude de la structure d'un réel lui-même construit. 
4.

Je reviens à la notion de la théorie physique. II est assez évident que le formalisme logico-mathématique n'engendre pas sa propre interprétation, l'homomorphisme $\varphi$ : $A_{p} \rightarrow M_{p}$ n'étant pas un isomorphisme. La question des applications empiriques s'élargit considérablement dans cette perspective : l'empirie physique se trouve ici imprégnée de théorie, informée de théorie. Je parlerai d'indétermination de l'empirique : l'empirique n'est pas univoque pas plus que le théorique ou le théorétique, ou plutôt le théorétique recouvre l'empirique, le transite, l'embrasse, le déborde. Le problème de l'applicabilité des mathématiques au monde physique se dissipe lorsqu'on insiste sur la multiplicité des modèles possibles ou la non-catégoricité de la théorie physique, c'est-à-dire son caractère non univoque. À ce moment-là, c'est la prolifération des théories et des modèles qui révèle le caractère constructif de la théorisation, son caractère approximatif, itératif : la théorie est en marche vers une meilleure théorie, non pas vers un réel déjà là, qui attendrait dans sa pudeur virginale qu'on le dévoile - le physicien français $B$. d'Espagnat parle d'un réel voilé. II y a donc progrès dynamique des théories; un bon exemple est la théorie quantique des champs qui conjugue mécanique quantique et relativité : je dis progrès, je devrais dire intégration dynamique. L'électromagnétisme de Maxwell intégré dans la mécanique relativiste, la mécanique statistique qui intègre thermodynamique de l'équilibre (pas celle de Prigogine qui n'existe pas encore) et mécanique classique, théorie quantique qui intègre comme cas-limite macroscopique la mécanique classique, ce sont tous là des exemples de progrès ou d'intégration dynamique des théories physiques, quoi qu'en veuille un Kuhn avec sa thèse de l'incommensurabilité. Les théories unifiées des champs en sont encore un bel exemple avec la théorie de l'unification du champ électromagmétique et du champ leptonique faible due à Weinberg et renormalisée 
par 'thooft. Tout cela est le cœur même de la structure dynamique des théories physiques. L'unification est progrès, je pense que c'est là la grande leçon de la physique. Mais il n'y a pas de grande théorie finale, ce n'est là qu'un vœu philosophique qui n'a de valeur que comme mythe : l'unité de la science ou du savoir est une unité toujours recommencée.

Yvon Gauthier

Département de philosophie

Université de Montréal 